\title{
An Energy and Area Efficient yet High-Speed Square-Root Carry Select Adder Structure
}

\author{
Milad Bahadori ${ }^{1}$, Mehdi Kamal ${ }^{1}$, Ali Afzali-Kusha ${ }^{1}$, Massoud Pedram ${ }^{2}$ \\ ${ }^{1}$ School of Electrical and Computer Engineering, University of Tehran, Tehran, Iran \\ ${ }^{2}$ Department of Electrical Engineering, University of Southern California, Los Angeles, CA, USA \\ \{milad.bahadori, mehdikamal, afzali\}@ut.ac.ir, pedram@usc.edu
}

\begin{abstract}
In this paper, we propose a modified carry select adder (CSLA) structure which is more power/energy and area-efficient compared to the existing CSLAs. The higher efficiency is achieved by modifying the logic formulations of the carry generation and selection (CGS) scheme and merging all of its redundant logic operations in the carry generation (CG) and carry selection (CS) units of the CGS-based CSLA (CGS-CSLA) structure. This leads to a simplified structure. Next, the proposed CSLA structure is employed to design efficient square-root CSLA (SQRT-CSLA) structure. The efficiency of the proposed SQRT-CSLA is investigated by comparing its speed, power, energy, and area parameters with those of some other SQRT-CSLA structures, including conventional SQRT-CSLA, binary to excess-1 converter, common Boolean logic, and the CGS-based SQRT-CSLA structures. The investigation, which is performed using HSPICE simulations based on a 45-nm bulk CMOS technology, includes 8, 16, 32, and 64-bit adder structures. The impact of voltage scaling on the efficiency of the proposed structure is also studied by changing the supply voltage levels from the near-threshold voltage to the nominal supply voltage. Simulation results reveal that the proposed SQRT-CSLA provides, at least, $14 \%, 14 \%$, and $15 \%$ lower energy, energydelay product, and area-delay product, respectively, compared to those of the CGS-based SQRT-CSLA structure, averaged over the supply voltage and bit length.
\end{abstract}

Keywords-Square-root carry select adder, carry generation and selection, energy and area efficient, supply voltage scaling.

\section{Introduction}

Being a key building block of arithmetic and logic units (ALUs) used in various microprocessors, it is highly desirable to lower the delay, power or energy consumptions, and area usage of adders [1], for efficient 
implementation of different applications such as signal processing applications [2, 3, 4, 5]. On the other hand, obtaining high speed at low energy and area consumptions is a challenge for designers of digital circuits [6, 7]. Supply voltage scaling is one of the most effective methods for lowering the energy per operation of circuits due to the quadratic dependence of the switching energy on the voltage $[8,9]$. Note also that the subthreshold current as the main leakage power component of the OFF CMOS transistors, has an exponential dependence on the supply voltage level through the drain induced barrier lowering (DIBL) effect $[8,9]$. The voltage downscaling, however, adversely affects the speed of the circuit. The dependence of the total power consumption and circuit speed on the supply voltage level has been the main motivation behind the development of dynamic voltage and frequency scaling techniques for digital very-large-scale integration (VLSI) circuits. In these circuits, to reduce the energy consumption, the system may change the voltage (and frequency) of the circuit based on the workload requirement [10]. For these systems, the circuit should be able to operate under a wide range of supply voltage levels. Recently, dynamic voltage scaling from the superthreshold voltage regime (i.e., the nominal supply voltage level) down to the near-threshold voltage regime (i.e., supply voltage levels near the threshold voltage of transistors) has been considered as an approach that provides a more desirable tradeoff point between delay (frequency) and energy consumption of the circuits $[8,9,10,11]$.

There are different adder families/structures where each has its own speed versus power/energy tradeoff. Also, these adders have different area usages. Among them, ripple carry adder (RCA) has the simplest structure leading to the smallest area and power consumption at the price of having the worst critical path delay [1,6]. Carry select adder (CSLA) and carry lookahead adder (CLA) structures alleviate the problem of the carry propagation delay of the RCA at the cost of increasing the power and area $[6,12]$. The former provides higher speed than that of the RCA and lower area than that of CLA [6]. The CSLA, however, suffers from the high power/energy consumption (i.e., power dissipation and power-delay product) due to the logic duplication in its structure $[12,13]$.

The focus of this work is on the CSLA family whose conventional structure contains two independent RCA blocks. Initially, using the blocks, the partial sums and carries are in parallel for both carry inputs of zero and one (i.e., $C_{\mathrm{i}}=0$ and $C_{\mathrm{i}}=1$ ). Next, the multiplexers select the appropriate final-sum and final-carry outputs based on the actual carry (see Fig. 1) $[6,14]$. A few prior works have attempted to improve performance parameters of the conventional CSLA structure by avoiding the logic duplication in the structure. Generally, these works may be classified into three categories. 


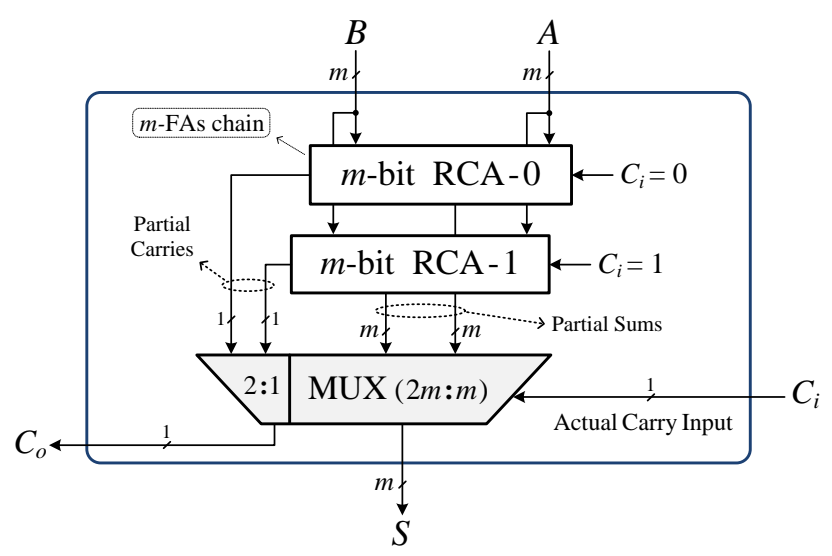

Fig. 1. Conventional structure of the CSLA [14].

The first category of techniques includes the works that utilize one RCA block with $C_{\mathrm{i}}=0$ and also, a modified circuit for computing the partial sum and carry corresponding to $C_{\mathrm{i}}=1$ based on the generated partial sum of the RCA block, instead of using two independent RCA blocks [15, 16, 17, 18] as detailed next. In [15] and [16], the CSLA structures were proposed which utilized a single RCA and a multiplexer-based add-one circuit instead of using two RCAs. The area usage and speed of this structure are lower than those of the conventional structure. To improve this adder, the add-one circuit presented in [17] was used to lower the area and delay of a 64-bit square-root CSLA (SQRT-CSLA) [19]. In a SQRT-CSLA structure, CSLAs with increasing sizes are connected in a cascading way. In this structure, the overall critical path delay of the adder is reduced by providing a parallel path for the carry propagation [1, 19]. In [18], instead of the RCA with $C_{\mathrm{i}}=1$ in the conventional CSLA, a binary to excess-1 converter (BEC) was used to lower the area usage and power consumption. The structure, however, has a lower speed compared to that of the conventional one.

The second category consists of works focusing on the modification of conventional CSLA structure by sharing the common Boolean logic (CBL) terms. In [20] and [21], by applying this method (i.e., logic simplification and sharing partial circuit) on the overall structure of the conventional CSLA, the area usage is significantly reduced at the cost of a considerable delay increase.

Finally, in the third category of techniques, the data dependency and redundant logic operations are identified based on analyzing logic operations in the previous CSLA structures. In [22], based on expressions for the logical operation, a carry generation and selection (CGS) scheme for the CSLA has been proposed. The CGS-based CSLA (CGS-CSLA) [22] has lower energy consumption and area-delay product (ADP) figures compared to those of the other CSLA structures. In the proposed CGS-CSLA structure of [22], however, not all of the redundant logic 
operations in different sub-blocks have been eliminated.

In this paper, we focus on reducing the power consumption and area usage further by eliminating all redundant logic operations in the internal structure of the CGS-CSLA without sacrificing the adder speed. The proposed modification is performed by optimizing and merging logic units of the carry generation (CG) and carry selection (CS) stages in the CGS-CSLA. The proposed scheme yields lower wiring tracks and structural complexity. Also, an efficient square-root CSLA structure based on cascading the modified CSLA structures is presented. Thus, the contributions of this work are itemized in the following:

1) Proposing a modified CSLA structure by merging the logic operations of the CGS scheme of the CGS-CSLA and eliminating all redundant operations in its structure so as to enhance the energy/area-efficiency of the adder.

2) Presenting the structure of the SQRT-CSLA based on the proposed CSLA structure.

3) Investigating the impact of supply voltage scaling on the efficiency of the CSLA structures.

The rest of this paper is organized as follows. Section II describes the overall structure of CGS-CSLA. The details of the proposed modified CSLA structure as well as the SQRT-CSLA structure by employing the proposed CSLA block are presented in Section III. The results of comparing the characteristics of the proposed structure with those of other CSLA structures are discussed in Section IV. Finally, the conclusion is drawn in Section V.

\section{CGS-CSLA Structure}

In this section, we present the overall structure and some background details about the conventional CGS-CSLA. The structure of an $m$-bit CGS-CSLA is shown in Fig. 2 [22]. It consists of a half-sum/carry generation (HSCG) unit, a carry generation (CG) unit, a carry selection (CS) unit, and a full-sum generation (FSG) unit. The HSCG unit receives two $m$-bit inputs (i.e., $A$ and $B$ ) and generates $m$-bit half-sum $(H S)$ and $m$-bit half-carry $(H C)$ words, using $m$ two-inputs exclusive-or (XOR) and $m$ two-inputs AND gates in parallel, respectively. The CG unit is composed of the $\mathrm{CG}_{0}$ and $\mathrm{CG}_{1}$ sub-blocks corresponding to $C_{\mathrm{i}}=0$ and $C_{\mathrm{i}}=1$, respectively. Both the $\mathrm{CG}_{0}$ and $\mathrm{CG}_{1}$ receive $H S$ and $H C$ from the HSCG unit and generate two $m$-bit full-carry words $F C_{0}$ and $F C_{1}$ corresponding to $C_{\mathrm{i}}=0$ and $C_{\mathrm{i}}=1$, respectively. Next, the CS unit selects the final $m$-bit full-carry word $(F C)$ based on the actual carry input $\left(C_{i}\right)$, where the most significant bit (MSB) of $F C$ (i.e., $F C(m))$ represents the carry output $\left(C_{o}\right)$ of the structure. In the final step, the FSG unit computes the final $m$-bit full-sum (FS) output using one bit $C_{i}$ and $(m-1)$-bit $F C$ as well as $m$ bit $H S$ by using $m$ parallel two-inputs XOR gates. 


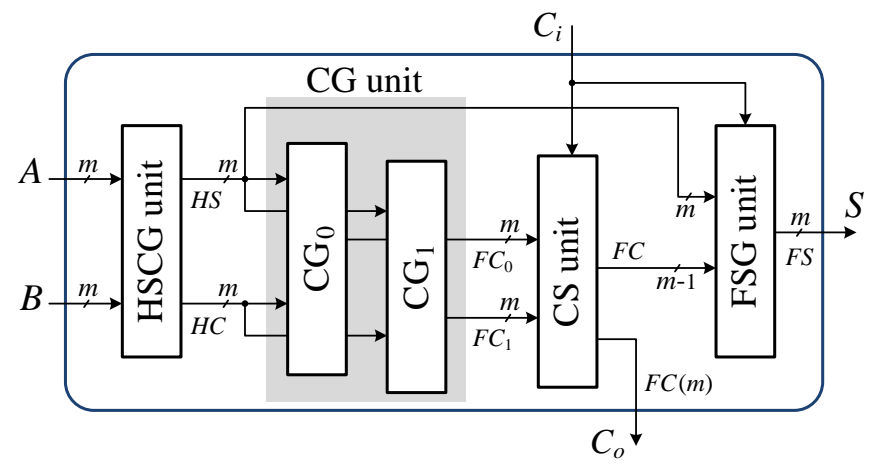

Fig. 2. Overall structure of the CGS-CSLA [22].

Based on the above explanation, in the CGS-CSLA structure, the CG and CS units are scheduled before the final sum output computation, which is different from the conventional CSLA method [22]. The internal structures of the CG and CS units are constructed based on the following logic expressions [22]:

$H S(k)=A(k) \oplus B(k) \quad ; \quad H C(k)=A(k) \cdot B(k)$

$F C_{0}(k)=F C_{0}(k-1) \cdot H S(k)+H C(k) ; \quad$ for $\left\{F C_{0}(0)=0\right\}$

$F C_{1}(k)=F C_{1}(k-1) \cdot H S(k)+H C(k) ; \quad$ for $\left\{F C_{1}(0)=1\right\}$

$F C(k)=\left\{\begin{array}{lll}F C_{0}(k) & \text { if } & \left(C_{i}=0\right) \\ F C_{1}(k) & \text { if } & \left(C_{i}=1\right)\end{array}\right.$

where $1 \leq k \leq m$ in the above relations and, $A(k)$ and $B(k)$ represent the $k^{\text {th }}$ bit of the $m$-bit $A$ and $m$-bit $B$, respectively. Notice that the $m$-bit sum output is computed by the following logic expression:

$F S(k)=H S(k) \oplus F C(k-1) ; \quad$ for $\quad 1 \leq k \leq m$

where $F C(0)=C_{i}$ and $F C(m)=C_{o}$. For more details about the structure of the CGS-CSLA, the interested reader may refer to [22].

\section{Proposed Modified CSLA Structure}

In this section, we first, describe our proposed CSLA scheme, next, the structure of the SQRT-CSLA based on the proposed CSLA structure is presented.

\section{A. Proposed CSLA structure}

The proposed structure is obtained by modifying the CGS-CSLA structure of [22]. Compared to the original structure, all the redundant logic operations are omitted to reduce the power consumption and area usage. The speed of the adder in the proposed structure, which we denote by IRCGS-CSLA (which stands for irredundant CGS- 
CSLA), is not lowered. The modification is performed by merging some logic operations of the CG and CS units in the CGS-CSLA structure.

Now, we describe the internal structure of the proposed modified CSLA. As mentioned in Section II, two independent $\mathrm{CG}_{0}$ and $\mathrm{CG}_{1}$ sub-blocks are used in the CG unit of the CGS-CSLA structure to generate full-carry words corresponding to $C_{\mathrm{i}}=0$ and $C_{\mathrm{i}}=1$, respectively, and in the next unit (i.e., CS unit), the appropriate carry word is selected based on the carry input signal. One may avoid using two independent carry generation blocks (corresponding to carry input ' 0 ' and ' 1 ') in the CG unit by eliminating the redundant logic operations of the two carry generation blocks. This is achieved by merging their operations with the logic operations of the CS unit as is explained next.

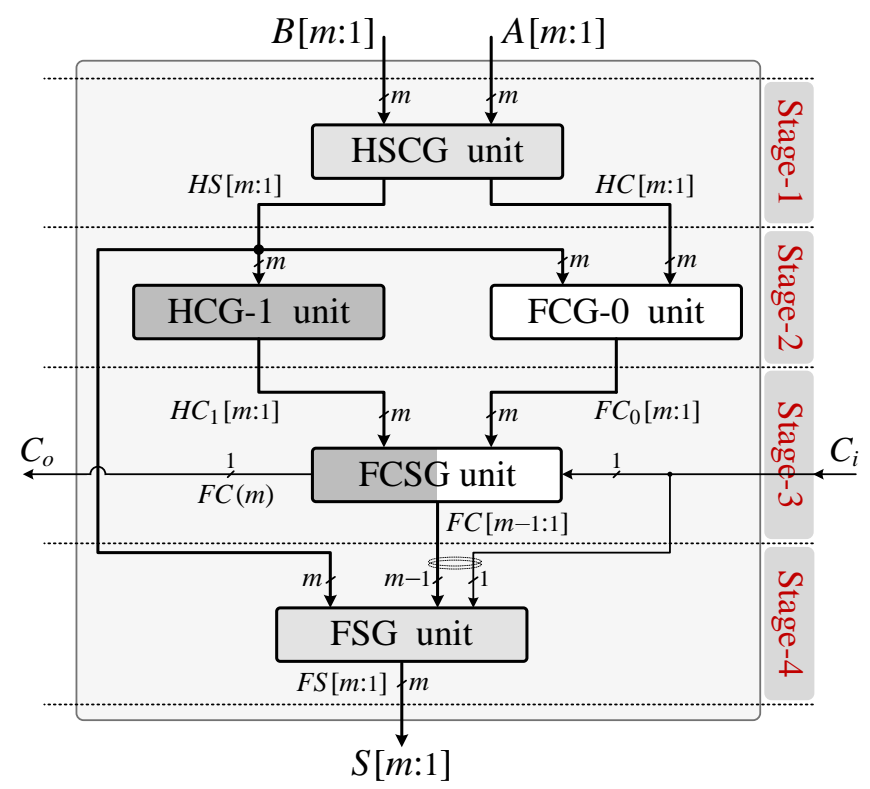

Fig. 3. Proposed IRCGS-CSLA structure.

The structure of an $m$-bit proposed IRCGS-CSLA, which contains four stages, is shown in Fig. 3. In the first stage, the half-sum/carry generation (HSCG) unit takes two $m$-bit $A$ and $B$ operands as the inputs and subsequently generates $m$-bit $H S$ and $H C$ words (see (1)). The optimized internal structure of the HSCG unit, which consists of $m$ parallel half-adder (HA) cells, is shown in Fig. 5(a). The modification of the original structure is based on the idea of using stages 2 and 3 where operations of the $\mathrm{CG}_{0}, \mathrm{CG}_{1}$, and $\mathrm{CS}$ logic units, shown in Fig. 2, are merged. To describe this scheme, let us rewrite (3) based on the logic expression (2) as follows (see Fig. 4),

$F C_{1}(k)=F C_{0}(k)+\prod_{j=1}^{k} H S(j) ;$ for $1 \leq k \leq m$.

It should be noted that the logic expression (6) is derived by eliminating all common logic operations of (2) and (3) 
(see Fig. 4). As shown in Fig. 4, the logic expressions (2) and (3) are expressed for $1 \leq k \leq m$. Expressing the logic expression (3) for the step $m$ based on the same expression for the previous step (i.e., $(m-1)^{\text {th }}$ step) and (2) of the same step (i.e., $m^{\text {th }}$ step), recursively, one may obtain the logic expression (6). Now, we define an $m$-bit word called half-carry corresponding to the corresponding to the carry input ' 1 ' $\left(H C_{1}\right)$ where

$H C_{1}(k)=\prod_{j=1}^{k} H S(j) ; \quad$ for $\quad 1 \leq k \leq m$.

Thus, expression (6) may be rewritten as

$F C_{1}(k)=F C_{0}(k)+H C_{1}(k) ; \quad$ for $\quad 1 \leq k \leq m$

On the other hand, logic expression (4) for the CS unit (see Fig. 2) may be rewritten as

$F C(k)=F C_{0}(k) \cdot \overline{C_{i}}+F C_{1}(k) \cdot C_{i} ; \quad$ for $\quad 1 \leq k \leq m$

Replacing expression (8) into (9) results in

$F C(k)=\left\{F C_{0}(k) \cdot \overline{C_{i}}+F C_{0}(k) \cdot C_{i}\right\}+H C_{1}(k) \cdot C_{i}=F C_{0}(k)+H C_{1}(k) \cdot C_{i} ; \quad$ for $\quad 1 \leq k \leq m$

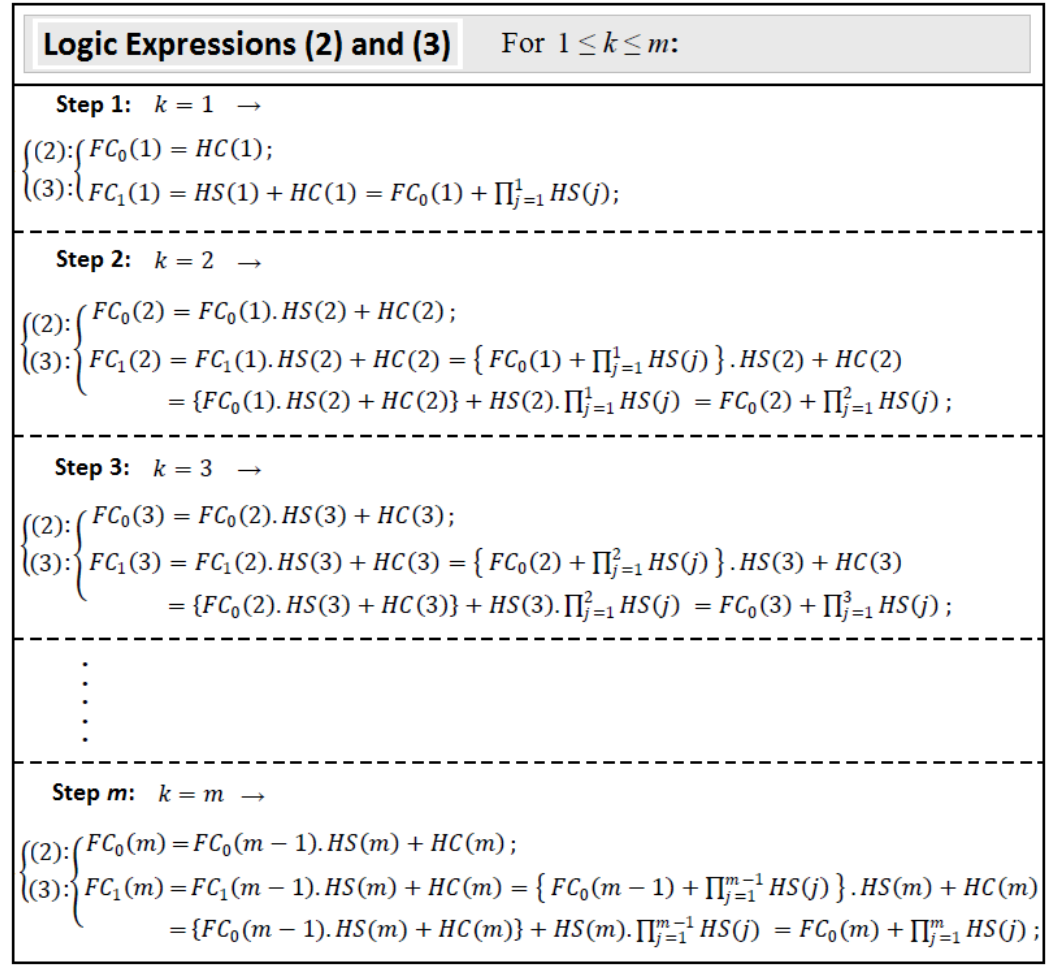

Fig. 4. The derivation of the logic expression (6) by eliminating all common logic operations of (2) and (3).

Consequently, instead of applying two independent carry generation sub-blocks (i.e., $\mathrm{CG}_{0}$ and $\mathrm{CG}_{1}$ ) in the $\mathrm{CG}_{\mathrm{unit}}$ of Fig. 2, we use a logic unit to compute $m$-bit $F C_{0}$ (denoted as the FCG-0 unit in Fig. 3) and another logic unit to 
compute $m$-bit $H C_{1}$ (denoted as the HCG-1 unit in Fig. 3). More precisely, the stage 2, called the CG stage, contains two logic units. The first one is a full-carry generation unit corresponding to $C_{i}=0$ (FCG-0 unit), which receives two $m$-bit $H S$ and $H C$ words and computes $m$-bit $F C_{0}$ based on (2). The second one is a half-carry generation unit corresponding to $C_{i}=1$ (HCG-1 unit), which receives only one $m$-bit $H S$ word and computes $m$-bit $H C_{1}$ using (see expression (7))

$H C_{1}(k)=H S(k) \cdot H C_{1}(k-1) ; \quad$ for $\quad\left\{H C_{1}(0)=1\right\}$

where $1 \leq k \leq m$. The optimized internal structures of the FCG-0 and HCG-1 units are shown in Fig. 5(b) and Fig. $5(\mathrm{c})$, respectively.

Now then, based on the modification of the CG unit of Fig. 2, the CS unit of the same figure must be also modified to perform the correct logic operations of the CGS scheme. More clearly, the CS unit must select $F C_{0}$ as the final $F C$, when $C_{i}$ is equal to zero whereas the CS unit must select $H C_{1}$ which is used for generating $F C_{1}$ as the final $F C$ when $C_{i}$ is equal to one. In this structure, the CS unit is responsible for compensating the reduced operations (complexity) of the $\mathrm{CG}$ unit (i.e., $\mathrm{CG}_{1}$ ). All of these operations are realized by the simple logical expression of (10). Based on (10), when $C_{i}$ is equal to zero (one), $F C$ will be $F C_{0}\left(F C_{0}+H C_{1}\right)$. Since the modified CS unit is organized based on both carry selection and carry generation operations, we use a full-carry selection/generation unit (denoted as the FCSG unit in Fig. 3) in the stage 3 of the proposed structure. The internal structure of the FCSG unit is shown in Fig. 5(d). This unit receives two $m$-bit $F C_{0}$ and $H C_{1}$ words as well as one bit $C_{i}$ as the inputs and generates an $m$-bit $F C$ as the output. The $m^{\text {th }}$ bit of the $F C$ (i.e., $\left.F C(m)\right)$ represents the carry output $\left(C_{o}\right)$ of the proposed structure. Merging the operations of the CG and CS units in the stages 2 and 3 enables us to reduce the required logic resources and wiring tracks of the CGS stage. For instance, the HCG-1 unit only has one $m$-bit input, simple structure, and smaller logic resources or the FCSG unit makes use of an optimized and simplified realization. Finally, in the stage 4 , the FSG unit computes the final $m$-bit $F S$ output using one bit $C_{i}$ and (m-1)-bit $F C$ as well as $m$-bit $H S$ based on the logic expression (5), cf. the FSG unit in Fig. 3. The internal structure of this stage is shown in Fig. 5(e). It should be noted that the critical delay paths of these logic units are illustrated by dashed lines in Fig. 5.

Now, to compare design parameters of IRCGS-CSLA and CGS-CSLA, we have provided the gate level netlist (based on two-input AND, OR, and XOR gates) of these two structures for the input bit length of 4 in Fig. 6 . It shows a gate count of 29 for the IRCGS-CSLA structure compared to 33 for the CGS-CSLA structure (more than 12 
$\%$ reduction). Also, eliminating all the redundant logic operations of the conventional structure, the complexity of the wiring (and wiring tracks) in the IRCGS-CSLA structure become lower than those of the CGS-CSLA implementation (see Fig. 6). In addition, the fan-outs of the intermediate nodes (and the corresponding capacitances) in both implementations are about the same.

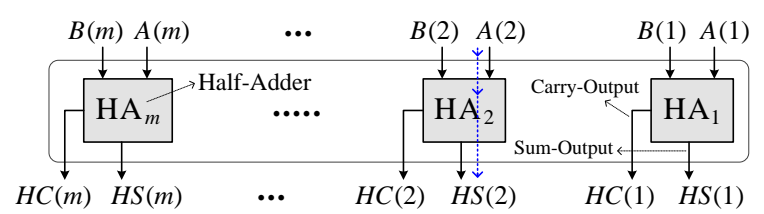

(a)

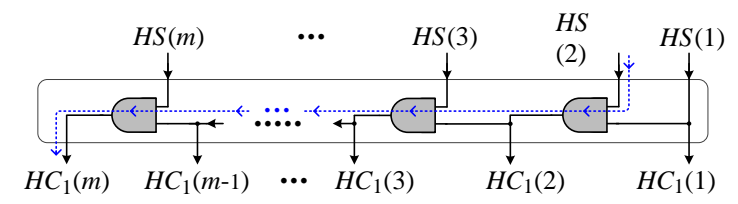

(c)

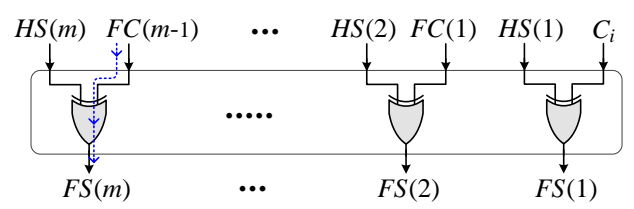

(e)

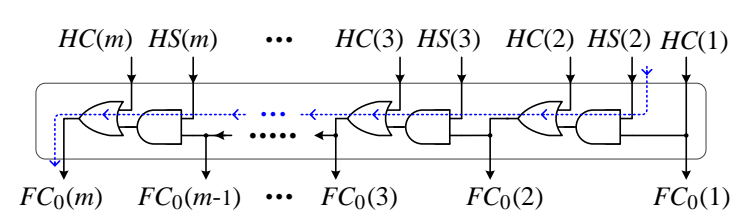

(b)

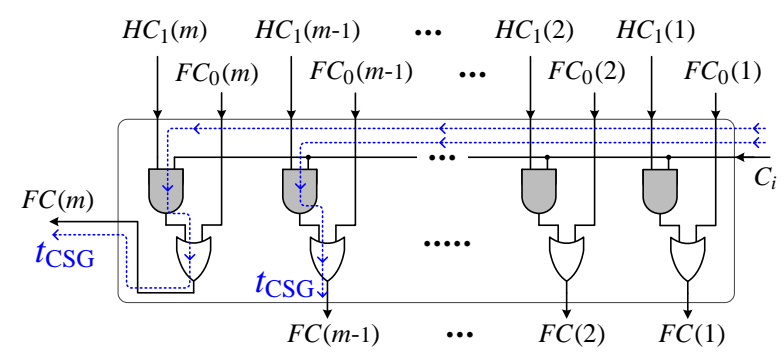

(d)

Fig. 5. Internal structures of the (a) HSCG, (b) FCG-0, (c) HCG-1, (d) FCSG, and (e) FSG units in the proposed IRCGS-CSLA.

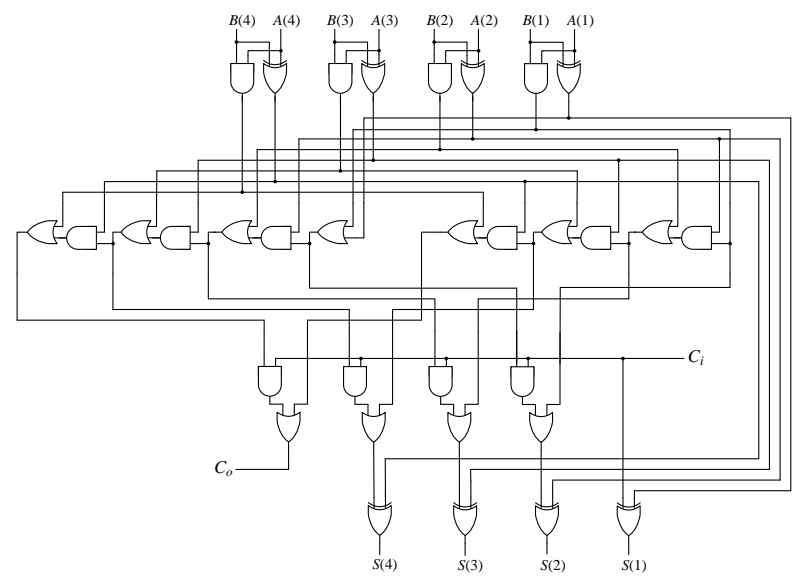

(a)

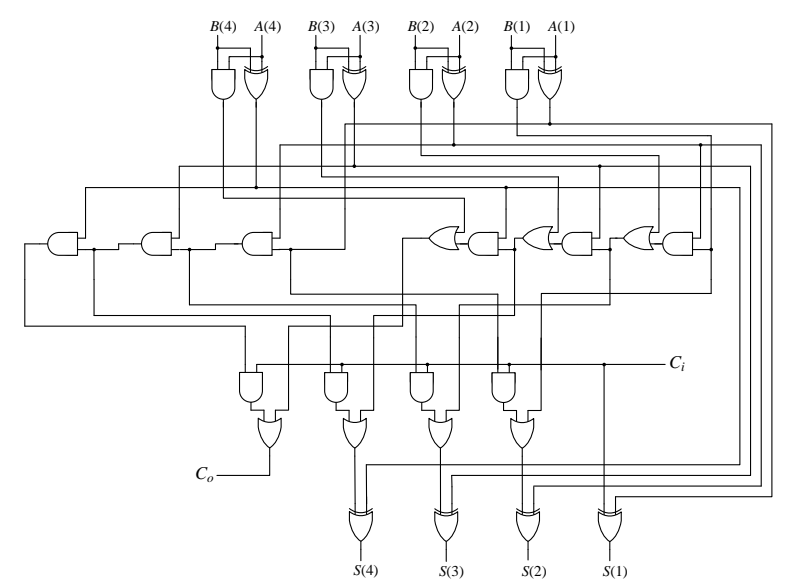

(b)

Fig. 6. Gate level implementations of 4-bit (a) CGS-CSLA, and (b) IRCGS-CSLA structures.

\section{B. Structure of SQRT-CSLA Based on the IRCGS-CSLA}

The basic idea behind developing SQRT-CSLA structure was to exploit multi-path carry propagation feature of 
the CSLA to improve the speed of the adder [19]. As mentioned before, in the SQRT-CSLA, the CSLAs with increasing sizes are connected in a cascaded structure. Sizes of the stages are selected by trying to nearly balance delays of the parallel paths. This way, large size adders may be implemented with a considerably less critical path delay than that of the corresponding single-stage CSLA [19, 22].

Fig. 7 shows the architecture of an $N$-bit SQRT-CSLA realized using the IRCGS-CSLA block. The structure contains $Q$ cascaded stages where the first stage is an $M_{1}$-bit RCA block (a full-adders (FAs) chain). From the second stage to the last stage, the IRCGS-CSLA blocks with increasing size are connected in a cascading configuration, while $M_{j} \geq M_{j-1}$ for $2 \leq j \leq Q$. The critical path of the proposed SQRT-CSLA contains three parts: 1) the path of the FA chain of the first stage whose delay is equal to the $t_{1}=M_{1} \times t_{\mathrm{CARRY}} ; 2$ ) the path of the intermediate carries selection/generation by the FCSG units whose delay is equal to the $(Q-1) \times t_{\mathrm{CSG}}=(Q-1) \times\left(t_{\mathrm{AND}}+t_{\mathrm{OR}}\right)$; and 3) the path of the final sum output generation by the FSG unit of the last stage whose delay is equal to the $t_{\mathrm{XOR}}$. Note that $t_{\mathrm{CARRY}}, t_{\mathrm{AND}}, t_{\mathrm{OR}}$, and $t_{\mathrm{XOR}}$ are the propagation delays of the carry output of an FA, two-inputs AND, OR, and XOR gates, respectively. Also, $t_{\mathrm{CSG}}$ is the critical path delay of the FCSG block, as shown in Fig. 5(d). Hence, the critical path delay $\left(T_{D}\right)$ of the proposed SQRT-CSLA is formulated by

$T_{D}=M_{1} \times t_{\mathrm{CARRY}}+(Q-1) \times\left(t_{\mathrm{AND}}+t_{\mathrm{OR}}\right)+t_{\mathrm{XOR}}$.

In the structure of the SQRT-CSLA, determining the stage sizes (i.e., bit-lengths of the stages) is very important for minimizing the critical path delay of the adder. Hence, one may use (12) to determine the size of the RCA block in the first stage (i.e., $M_{1}$ ) and the number of stages (i.e., $Q$ ) in designing SQRT-CSLA.

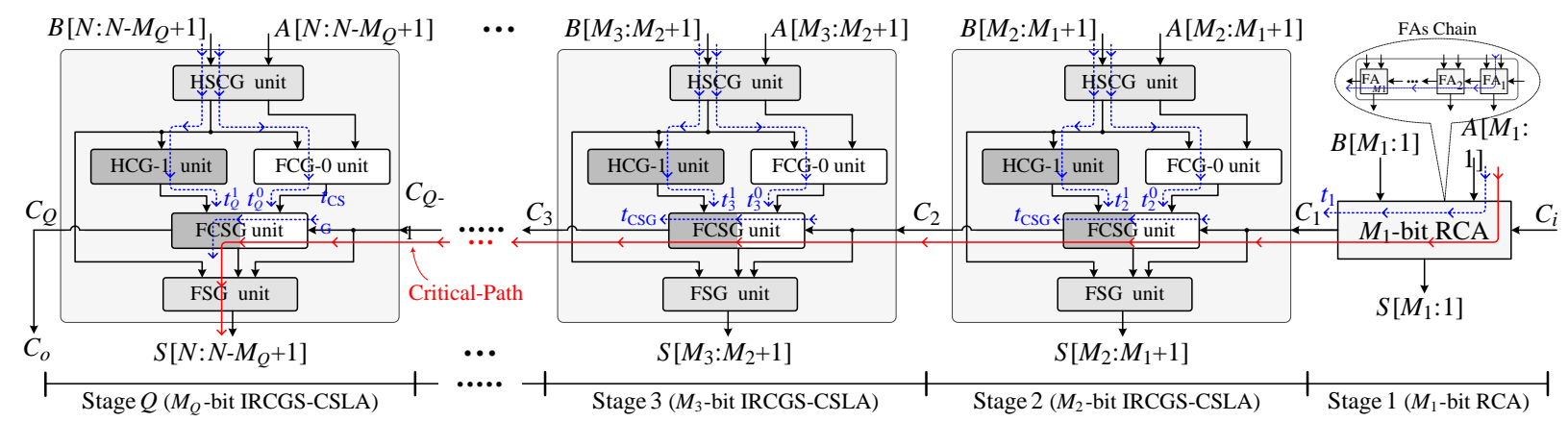

Fig. 7. Architecture of the proposed SQRT-CSLA based on the IRCGS-CSLA structure.

\section{Results and Discussion}

In this section, we assess the efficacy of the proposed SQRT-CSLA structure by comparing its delay, power, 
energy, and area with those of other SQRT-CSLA structures for different bit-lengths using a 45-nm static CMOS standard cells library [23]. In the first step, the optimum stage sizes of the adders, in the case of IRCGS-CSLA (IRCGS-SQRT-CSLA), were obtained based on the considered standard cells library. The sizes of the stages were \{1-bit RCA and 2-, 2-, and 3-bit IRCGS-CSLAs\}, \{2-bit RCA and 2-, 3-, 4-, and 5-bit IRCGS-CSLAs\}, \{2-bit RCA and 2-, 3-, 3-, 4-, 5-, 6-, and 7-bit IRCGS- CSLAs\}, and \{2-bit RCA and 2-, 3-, 4-, 5-, 6-, 7-, 8-, 8-, 9- and 10-bit IRCGS-CSLAs\} for the 8-, 16-, 32-, and 64-bit SQRT-CSLA structures, respectively. The comparative studies also include the conventional SQRT-CSLA (Conv-SQRT-CSLA) [14], BEC-based SQRT-CSLA (BEC-SQRT-CSLA) [18], CBL-based SQRT-CSLA (CBL-SQRT-CSLA) [20], and CGS-based SQRT-CSLA (CGS-SQRT-CSLA) [22] structures. Efficient 8-, 16-, 32-, and 64-bit length structures of these four SQRT-CSLAs were designed (at the gate level) based on their presented architectures and methods using the considered standard cells library.

In the second step, all the designs of the adders at the gate level were synthesized using the Synopsys Design Compiler tool [24] based on (typical) NanGate standard cell library for the 45-nm bulk CMOS technology [23]. The sizes of the standard cells in each structure were determined based on the constraint of high speed. Based on these sizes, the simulations for measuring delay, power, and energy parameters of the adders were performed using HSPICE [26]. The SPICE model of the standard cells of the NanGate library at the room temperature of $25{ }^{\circ} \mathrm{C}$ were exploited in these simulations. Finally, the netlist files obtained from the Design Compiler tool were imported to the Cadence SOC Encounter tool [25] to estimate the area usage of the implementations.

The nominal supply voltage of the considered technology was $1.1 \mathrm{~V}$ and the threshold voltages of the nMOS and pMOS transistors were $0.67 \mathrm{~V}$ and $-0.62 \mathrm{~V}$, respectively. It should be noted that, to extract the power consumption of the adders, 10,000 uniform random stimuli were injected to them. Also, for each adder structure at each supply voltage level, the injection rate of the stimuli was chosen based on the maximum operating frequency of the adder structure. In the two following subsections, we first focus on studying the effectiveness of the proposed 32-bit SQRT-CSLA structure operating under a wide range of supply voltage levels (from the nominal voltage down to the near-threshold voltage), and then investigate the impact of the bit-length on the efficiency of the proposed structure.

\section{A. SQRT-CSLA Structures Operating Under a Wide Range of Supply Voltage Levels}

In this subsection, the critical path delays, power consumptions, power-delay products (energies), and energydelay products of the adders are compared. The results were obtained for a wide range of supply voltage levels from the nominal voltage (superthreshold voltage) to the nMOS threshold voltage ( $\left.\mathrm{V}_{\mathrm{TH}, \mathrm{nMOS}}\right)$ (near-threshold voltage). 
The critical path delays of the adders versus the supply voltage are plotted in Fig. 8(a). As the results show, the critical path delays of the Conv-SQRT-CSLA, CGS-SQRT-CSLA, and IRCGS-SQRT-CSLA structures are almost similar and have the smallest delays under all the supply voltages. The CBL-SQRT-CSLA has the highest critical path delay which is about 3 times more than those of the other adders at all considered voltage levels. Also, the delay of the BEC-SQRT-CSLA is about 17\% to $19 \%$ larger than that of the proposed IRCGS-SQRT-CSLA for the considered voltage range. Finally, the results indicate that reducing the supply voltage from $1.1 \mathrm{~V}$ to the nMOS threshold voltage (i.e., $0.67 \mathrm{~V}$ ) causes an about 11.7 fold increase in the critical path delay for all the adders.

The power consumptions of the adders versus the supply voltage are shown in Fig. 8(b). The results reveal that the smallest power consumption belongs to the CBL-SQRT-CSLA while the Conv-SQRT-CSLA structure consumes the highest power owing to the duplication of the RCA blocks in its structure. After Conv-SQRT-CSLA, the BEC-SQRT-CSLA has the highest power consumption. As shown in Fig. 8(b), the power consumption of the CGS-SQRT-CSLA is more than the IRCGS-SQRT-CSLA while it is smaller than that of the BEC-SQRT-CSLA structure at all the supply voltages. For the supply voltages considered here, the power reductions of the proposed IRCGS-SQRT-CSLA structure compared with those of the CGS-SQRT-CSLA (Conv-SQRT-CSLA) were in the range of $13 \%$ to $14 \%$ ( $44 \%$ to $46 \%$ ). The power consumption of the IRCGS-SQRT-CSLA is about 1.8 times more than that of the CBL-SQRT-CSLA under the all considered supply voltage levels. Finally, the results reveal, on average, a 32 times reduction in the power consumption of the adders when scaling the supply voltage from $1.1 \mathrm{~V}$ (nominal voltage) to the nMOS threshold voltage (near-threshold voltage).

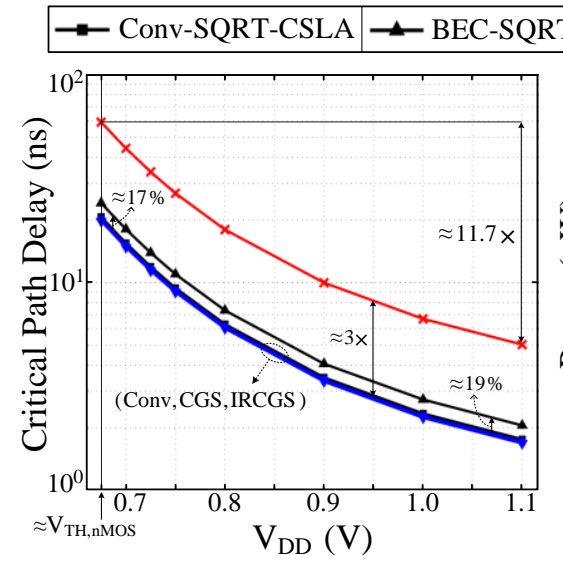

(a)

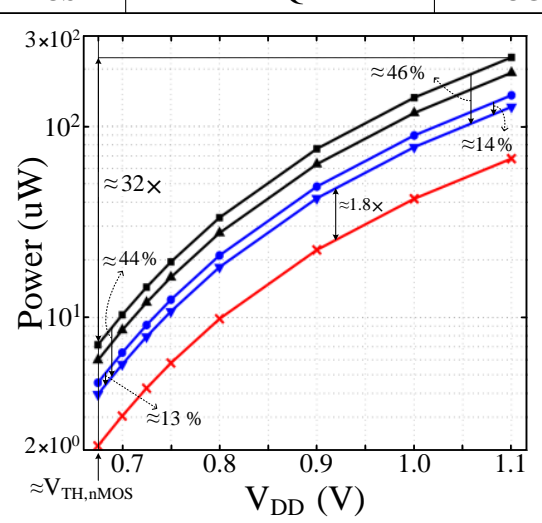

(b)

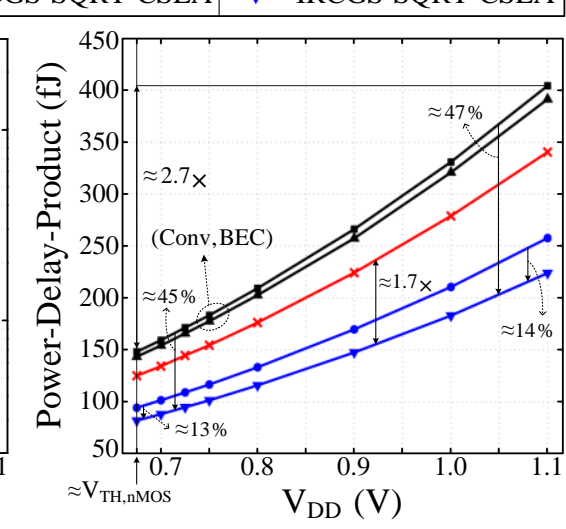

(c)

Fig. 8. (a) Critical path delay, (b) power consumption, and (c) power-delay product (energy) of the 32-bit adder structures versus the supply voltage. 
Fig. 8(c) shows the power-delay product (PDP) of the adders for different supply voltages. The proposed IRCGSSQRT-CSLA has the best PDP compared to those of the other structures in the supply voltage range considered in this work. The highest PDP corresponds to the Conv-SQRT-CSLA, and the PDP of the BEC-SQRT-CSLA is a little smaller than that of the Conv-SQRT-CSLA structure. Next to Conv-SQRT-CSLA and BEC-SQRT-CSLA structures, the CBL-SQRT-CSLA has the highest PDP which is about 1.7 times more than that of the proposed IRCGS-SQRT-CSLA. Also, the PDP of the CGS-SQRT-CSLA is smaller (larger) than that of the (CBL-SQRTCSLA) IRCGS-SQRT-CSLA. Additionally, the PDP values of the proposed IRCGS-SQRT-CSLA structure are about $13 \%$ to $14 \%$ ( $45 \%$ to $47 \%$ ) lower compared the corresponding values in the case of the CGS-SQRT-CSLA (Conv-SQRT-CSLA) structures. Finally, the results reveal that reducing the supply voltage from $1.1 \mathrm{~V}$ to the nMOS threshold voltage causes an about 2.7 fold decrease in the PDP for all the adders.

The values of the energy-delay product (EDP) of the adders versus the supply voltage are plotted in Fig. 9(a). The results show that the CBL-SQRT-CSLA has the largest EDP due to its lowest speed. Also, the proposed IRCGSSQRT-CSLA has the smallest EDP, due to its smaller power consumption as well as higher speed. In addition, the EDP of the CBL-SQRT-CSLA is about five times more than that of the proposed IRCGS-SQRT-CSLA under all the supply voltages. After the CBL-SQRT-CSLA, the BEC-SQRT-CSLA and the Conv-SQRT-CSLA structures have the highest EDPs, respectively. Furthermore, the EDP of the CGS-SQRT-CSLA is smaller (higher) than the ConvSQRT-CSLA (proposed IRCGS-SQRT-CSLA) structure. Finally, to demonstrate the trade-offs between the delay and energy for each adder structure, the energy-delay Pareto-optimal curves are plotted in Fig. 9(b) which suggests the proposed IRCGS-SQRT-CSLA structure as the better adder.

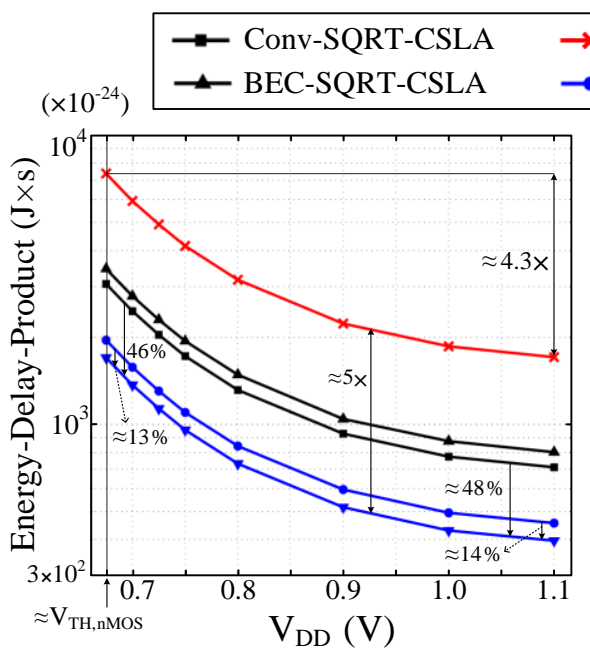

(a)

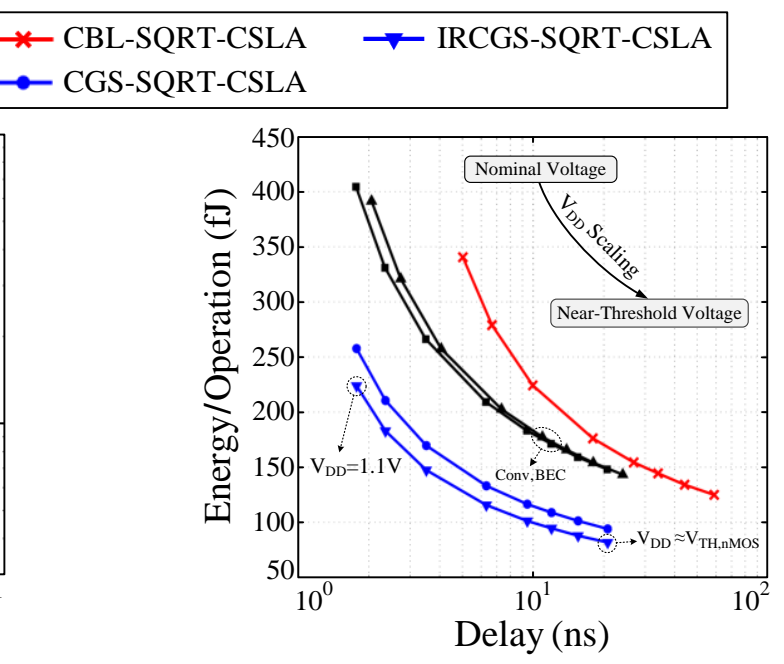

(b) 
Fig. 9. (a) Energy-delay-product of the adder structures versus the supply voltage. (b) Energy-delay Pareto-optimal curves for different adder structures.

Therefore, the proposed IRCGS-CSLA structure is a better candidate for high-speed and low-energy applications, compared with the existing CSLA structures. Note that such comparative studies have been performed for the 8-, 16and 64-bit SQRT-CSLA structures similar to the 32-bit structures. The results showed that the efficacies of the 8-, 16-, and 64-bit proposed IRCGS-SQRT-CSLA structures (in the delay and power/energy parameters) are almost similar to its 32-bit structure under all the supply voltages, compared with other SQRT-CSLA structures with the same bit-lengths. Hence, for the sake of space, in the next section, the impact of the bit-length on the efficiencies of the different square-root carry-select adder structures is performed at the nominal voltage (i.e., $\mathrm{V}_{\mathrm{DD}}=1.1 \mathrm{~V}$ ) and near-threshold voltage (i.e., $\mathrm{V}_{\mathrm{DD}} \approx \mathrm{V}_{\mathrm{TH}, \mathrm{nMOS}}$ ).

\section{B. Different Bit-Lengths of SQRT-CSLA Structures}

In this subsection, to study the impact of the bit-lengths on the efficiency of the adders, the area, PDP, EDP, and area-delay product (ADP) of the structures at the nominal and near-threshold voltages are compared. Table I reports the area usages of the SQRT-CSLAs for 8-, 16-, 32-, and 64-bit lengths. The Conv-SQRT-CSLA structure has the highest area for all the considered bit-lengths. The next largest adder is CBL-SQRT-CSLA (BEC-SQRT-CSLA) for 8-bit (16-, 32-, and 64- bit) length(s). For 8- and 16-bit (32- and 64-bit) adders, the IRCGS-SQRT-CSLA (CBLSQRT-CSLA) has the smallest area. The area usage of the proposed IRCGS-SQRT-CSLA structure is on average about 15\% smaller than that of the CGS-SQRT-CSLA structure. Thus, the results show that the proposed structure is an area-efficient CSLA compared to other CSLA structures, while having high-speed and energy efficiency features.

Table I Area Usages of the Different SQRT-CSLA Structures.

\begin{tabular}{|c|c|c|c|c|c|c|}
\hline & & \multicolumn{5}{|c|}{ SQRT-CSLA } \\
\hline & & Conv- & BEC- & CBL- & CGS- & IRCGS- \\
\hline \multirow{4}{*}{ Area $\left(\mu \mathrm{m}^{2}\right)$} & 8-bit & 82.2 & 67.5 & 74.3 & 52.7 & 44.3 \\
\hline & 16-bit & 172.5 & 138.8 & 102.8 & 108.3 & 91.1 \\
\hline & 32-bit & 357.4 & 281.3 & 162.0 & 218.7 & 186.0 \\
\hline & 64-bit & 726.8 & 565.3 & 318.7 & 438.2 & 376.6 \\
\hline
\end{tabular}

Fig. 10(a) and Fig. 10(b) show the PDP and EDP of the SQRT-CSLA structures, respectively. The results reveal that the proposed IRCGS-SQRT-CSLA structure has the best PDP and EDP compared with other SQRT-CSLAs for all three bit-lengths. Also, Fig. 10(c) shows the ADP of the SQRT-CSLA structures which indicate that the ADP of 
the proposed IRCGS-SQRT-CSLA is the lowest compared to those of the other structures. Note that the figure reveals that the power/energy saving and the area reduction slightly decrease with increasing the bit-length.

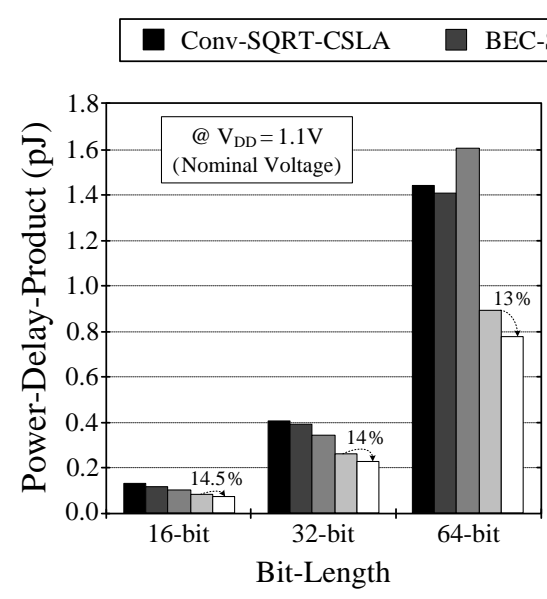

(a)

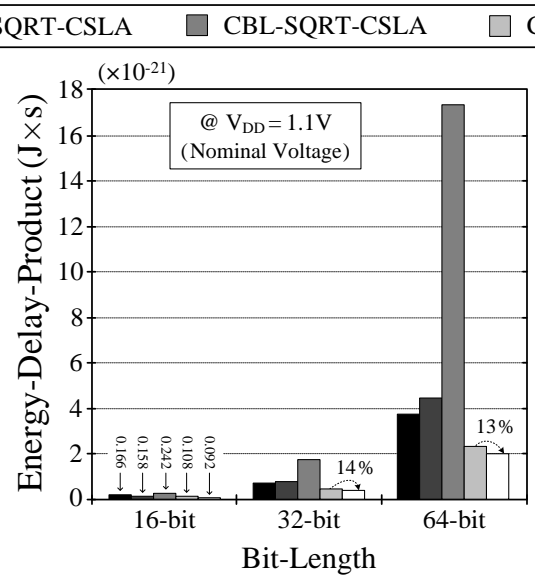

(b)

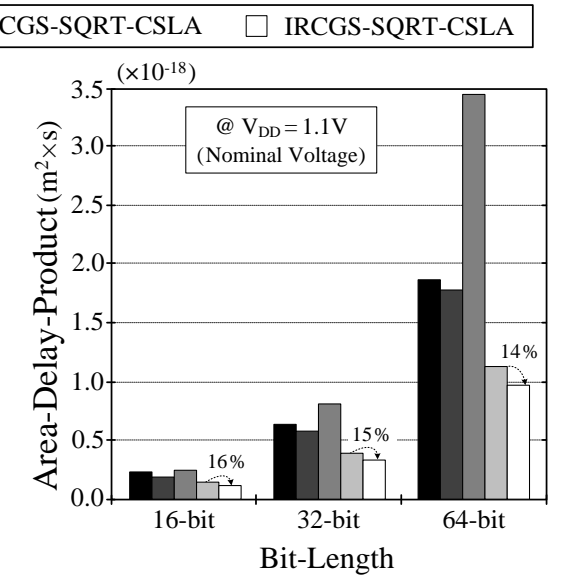

(c)

Fig. 10. Comparison of (a) power-delay product (PDP), (b) energy-delay product (EDP), and (c) area-delay product (ADP) of the adder structures at the nominal supply voltage for the different bit-lengths.

Since 8- and 16-bit adder designs dominate in low voltage (i.e., near-threshold voltage operation) consumer electronics products, therefore, the results for 8- and 16-bit SQRT-CSLA structures are presented in Fig. 11. The results include PDP, EDP, and ADP parameters of both 8-bit and 16-bit adder structures for both nominal and nearthreshold supply voltages. The results demonstrate superiority of the proposed IRCGS-SQRT-CSLA structure in terms of the design parameters of PDP, EDP, and ADP when compared to other adder structures at both the nominal and near-threshold voltages. Finally, it may be concluded that for all the four bit-lengths, the low delay (high-speed) of the proposed IRCGS-SQRT-CSLA structure along with its lower power/energy consumption and smaller area usage result in the minimum PDP, EDP, and ADP for this structure compared with the other existing SQRT-CSLA structures for both the superthreshold and near-threshold voltage regimes. 


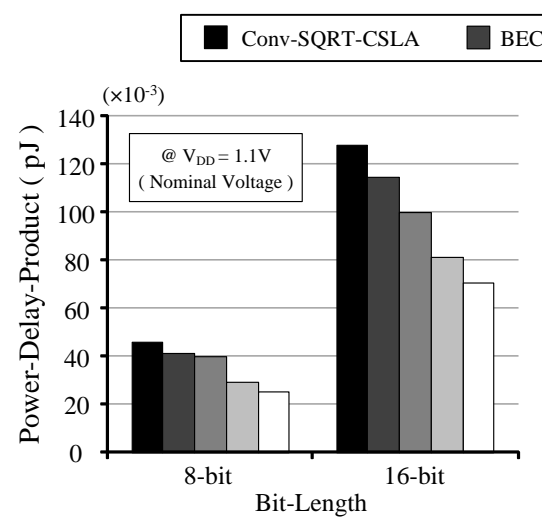

(a)

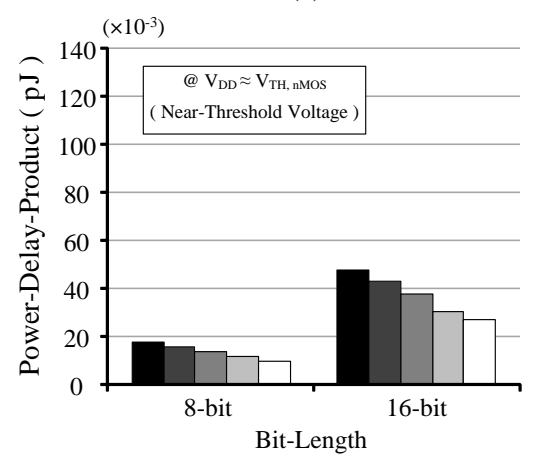

(d)

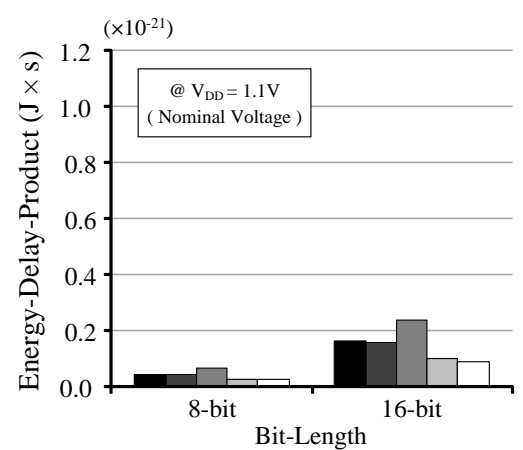

(b)

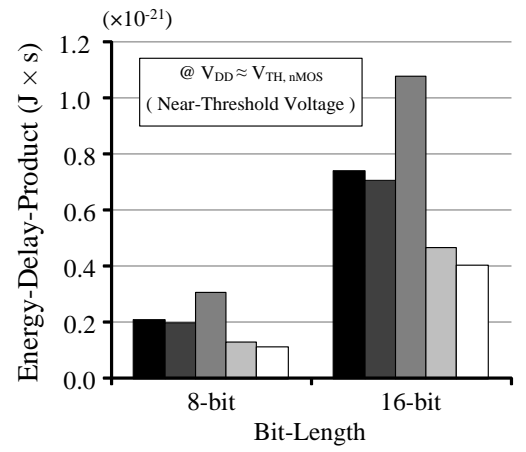

(e)

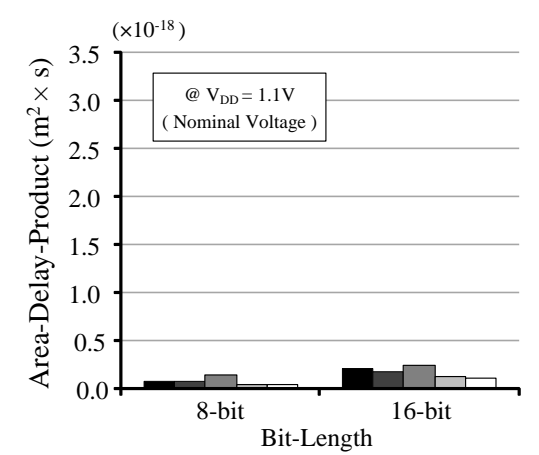

(c)

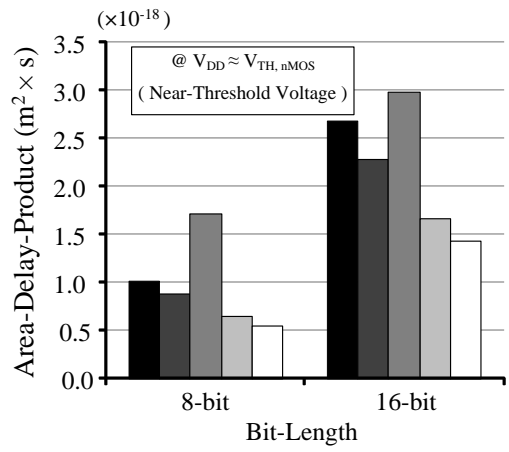

(f)

Fig. 11. Comparison of (a) PDP, (b) EDP, and (c) ADP of 8-bit and 16-bit adders for the nominal supply voltage and (d) PDP, (e) EDP, and (f) ADP of 8-bit and 16-bit adders for the near-threshold voltage.

\section{Conclusion}

In this paper, a modified CSLA structure which had a lower energy consumption compared to the existing CSLAs was proposed. The proposed adder was high-speed and area-efficient too. The improvements in the power/energy and area were achieved by merging the redundant logic operations in an efficient CSLA structure called CGSCSLA. The efficient CGS scheme uses optimized carry generation (CG) and carry selection (CS) units. Based on this structure, we developed an efficient SQRT-CSLA structure by cascading the proposed CSLA blocks with increasing sizes. The efficacy of the proposed SQRT-CSLA structure (denoted by IRCGS-SQRT-CSLA) was compared to those of the Conv-SQRT-CSLA, BEC-SQRT-CSLA, CBL-SQRT-CSLA, and CGS-SQRT-CSLA structures for different bit-lengths under a wide range of supply voltage scaling, from the nominal supply voltage (superthreshold voltage) down to the near-threshold voltage. The suggested high-speed adder structure showed the lowest PDP, EDP, and ADP making itself as a better candidate for high-speed low-energy applications. 


\section{Acknowledgement}

The work of M. Bahadori, M. Kamal, and A. Afzali-Kusha was supported by the Iran National Science Foundation.

\section{References}

[1] I. Koren, Computer Arithmetic Algorithms, 2nd edition A. K. Peters, Ltd., Natick, MA, 2002.

[2] M. Kumar and T. K. Rawat, "Fractional order digital differentiator design based on power function and least squares,” International Journal of Electronics, Vol. 103, Issue 10, pp. 1-15, February 2016.

[3] M. Kumar and T. K. Rawat, "Design of a variable fractional delay filter using comprehensive least square method encompassing all delay values," Journal of Circuits, Systems and Computers, Vol. 24, Issue 08, pp. 155-116, September 2015.

[4] A. Aggarwal, T. K. Rawat, D. K. Upadhyay, and M. Kumar, "Efficient design of digital FIR differentiator using L $1^{-}$ method," RADIOENGINEERING, Vol. 25, No. 2, June 2016.

[5] A. Aggarwal, M. Kumar, T. K. Rawat, and D. K. Upadhyay, "Optimal design of 2D FIR filters with quadrantally symmetric properties using fractional derivative constraints," Circuits, Systems, and Signal Processing, Vol. 35, Issue 6, pp.2213-2257, June 2016.

[6] R. Zimmermann, "Binary adder architectures for cell-based VLSI and their synthesis," Ph.D. dissertation, Dept. Inf. Technol. Elect. Eng., Swiss Federal Inst. Technol. (ETH), Zürich, Switzerland, 1998.

[7] S. Ghosh, D. Mohapatra, G. Karakonstantis, and K. Roy, "Voltage scalable high-speed robust hybrid arithmetic units using adaptive clocking," IEEE Transactions on Very Large Scale Integration (VLSI) Systems, vol. 18, no. 9, pp. 1301-1309, September 2010.

[8] D. Markovic, C. C. Wang, L. P. Alarcon, L. Tsung-Te, J. M. Rabaey, "Ultralow-power design in near-threshold region," Proceedings of the IEEE, vol. 98, no. 2, pp. 237-252, February 2010.

[9] R. G. Dreslinski, M. Wieckowski, D. Blaauw, D. Sylvester, and T. Mudge, "Near-threshold computing: Reclaiming Moore's law through energy efficient integrated circuits," Proceedings of the IEEE, vol. 98, no. 2, pp. 253-266, February 2010.

[10] S. Jain et al., “A 280mV-to-1.2V wide-operating-range IA-32 processor in 32nm CMOS," IEEE International Solid-State Circuits Conference Digest of Technical Papers (ISSCC), pp. 66-68, 19-23 February 2012.

[11] M. Alioto, "Ultra-low power VLSI circuit design demystified and explained: A tutorial," IEEE Transactions on Circuits and Systems I (TCAS-I): Regular Papers, vol.59, no.1, pp.3-29, January 2012.

[12] V. G. Oklobdzija, B. R. Zeydel, H. Dao, S. Mathew, and R. Krishnamurthy, "Comparison of high-performance VLSI adders in energy-delay space,” IEEE Transactions on Very Large Scale Integration (VLSI) Systems, vol. 13, no. 6, pp. 754-758, June 2005.

[13] Y. He and C. H. Chang, "A power-delay efficient hybrid carry-lookahead/carry-select based redundant binary to two's complement converter," IEEE Transactions on Circuits and Systems I: Regular Papers, vol. 55, no. 1, pp. 336-346, February 2008.

[14] O. J. Bedrij, “Carry-select adder,” IRE Transactions on Electronic Computers, vol. EC-11, no. 3, pp. 340-346, June 1962.

[15] T.-Y. Chang and M.-J. Hsiao, “Carry-select adder using single ripple carry adder,” Electronics Letters, vol. 34, no. 22, pp. 2101-2103, October 1998. 
[16] Y. Kim and L. S. Kim, “64-bit carry-select adder with reduced area,” Electronics Letters, vol. 37, no. 10, pp. 614-615, May 2001.

[17] Y. He, C. H. Chang, and J. Gu, “An area-efficient 64-bit square root carry-select adder for low power application,” IEEE International Symposium on Circuits and Systems (ISCAS), vol. 4, pp. 4082-4085, 23-26 May 2005.

[18] B. Ramkumar and H.M. Kittur, "Low-power and area-efficient carry select adder," IEEE Transactions on Very Large Scale Integration (VLSI) Systems, vol. 20, no. 2, pp. 371-375, February 2012.

[19] M. Alioto, G. Palumbo, and M. Poli, “Optimized design of parallel carry-select adders," Integration, the VLSI Journal (Elsevier), vol. 44, Issue 1, pp. 62-74, January 2011.

[20] I.-C. Wey, C.-C. Ho, Y.-S. Lin, and C. C. Peng, "An area-efficient carry select adder design by sharing the common Boolean logic term," Proceedings on the International Multiconference of Engineering and Computer Scientist (IMECS), pp. 1-4, March 2012.

[21] S. Manju and V. Sornagopal, "An efficient SQRT architecture of carry select adder design by common Boolean logic," International Conference on Emerging Trends in VLSI, Embedded System, Nano Electronics and Telecommunication System (ICEVENT), pp.1-5, 7-9 January 2013.

[22] B. K. Mohanty and S. K. Patel, “Area-delay-power efficient carry-select adder," IEEE Transactions on Circuits and Systems II: Express Briefs, vol. 61, No. 6, pp. 418-422, June 2014.

[23] NanGate 45 nm Open Cell Library. [Online]. Available: http://www.nangate.com/, accessed December 2010.

[24] Synopsys Design Compiler. [Online]. Available: www.synopsys.com.

[25] Cadence SOC Encounter. [Online]. Available: http//www.cadence.com, version 10.1.

[26] Synopsys HSPICE. [Online]. Available: http://www.synopsys.com/, accessed September 2011.

Milad Bahadori received his M.Sc. and Ph.D. in Electrical Engineering from Sharif University of Technology, and University of Tehran in 2011, and 2015, respectively. His current research interests include digital embedded system design, approximate computing, low-power high-performance VLSI design, reliability in nanoscale design, nearthreshold computing and high-performance arithmetic circuits design.

Mehdi Kamal received his M.Sc. and Ph.D. in Computer Engineering from Sharif University of Technology, and University of Tehran in 2007, and 2013, respectively. He is currently the assistant professor with the School of Electrical and Computer Engineering of the University of Tehran. His research interests include digital embedded system design, approximate computing, neuromorphic computing and design for manufacturability.

Ali Afzali-Kusha received his Ph.D. in Electrical Engineering from University of Michigan in 1994. He is currently a Professor of the School of Electrical and Computer Engineering at the University of Tehran and the Director of Low-Power High-Performance Nanosystems Laboratory. He is a senior member of IEEE, and his current research interests include low-power high-performance design methodologies for nanoelectronics era.

Massoud Pedram is the Stephen and Etta Varra Professor of Electrical Engineering at the University of Southern California. He received his Ph.D. in EECS from UC-Berkeley in 1991. He is an IEEE Fellow, an ACM 
Distinguished Scientist, and past Editor-in-Chiefs of the ACM TODAES and IEEE JETCAS. His research focuses on energy-efficient computing, energy storage systems, and low power electronics. 